\title{
Research on Agricultural Surveillance Video of Intelligent Tracking
}

\author{
Lecai Cai*, Jijia Xu, Jin Liangping, and Zhiyong He \\ Institute of Computer Application, Sichuan University of Science and Engineering, \\ Zigong Sichuan, P.R. China 643000, \\ Tel.: +86-13508170118; Fax: +86-813-5505966 \\ clc@suse.edu.cn
}

\begin{abstract}
Intelligent video tracking technology is the digital video processing and analysis of an important field of application in the civilian and military defense have a wide range of applications. In this paper, a systematic study on the surveillance video of the Smart in the agricultural tracking, particularly in target detection and tracking problem of the study, respectively for the static background of the video sequences of moving targets detection and tracking algorithm, the goal of agricultural production for rapid detection and tracking algorithm and Mean Shift-based translation and rotation of the target tracking algorithm. Experimental results show that the system can effectively and accurately track the target in the surveillance video. Therefore, in agriculture for the intelligent video surveillance tracking study, whether it is from the environmental protection or social security, economic efficiency point of view, are very meaningful.
\end{abstract}

Keywords: video tracking, Mean-Shift algorithm, Epanechnikov core, density estimation.

\section{Introduction}

China's agricultural research, although the application of information technology is springing up in recent years, but the development is very fast. During the period of 1970s to 1980s, the State Science and Technology Commission, the Ministry of Agriculture has supported some crops expert system, decision support system, and agricultural production management system, Scientific and technological information networks, databases and other systems of research making some important results, many of them have been applied. Since 1996, the national "863" Project carried out. The application of intelligent agricultural information technology demonstration projects, research and development of a series of technical content was high, with independent intellectual property rights of agricultural technology should be service platform and a variety of practical tools, has established more than 100 agriculture applications. Intelligent expert system has a wide range in the domestic demonstration area from the original four provinces have developed to the 20 provinces. Therefore the research on agricultural surveillance video of intelligent tracking will bring us significant economic benefits.

\footnotetext{
* Corresponding author.
} 
The first concept of Mean Shift (Fukunaga et al,1975) is Fukunaga et. in 1975 in a density gradient with respect to the probability estimation function proposed by its original meaning, as its name implies, is to shift the mean vector, where is the Mean Shift a noun, it refers to a vector, however, with the development of Mean Shift Theory, the meaning of Mean Shift has changed, if we say that Mean Shift algorithm, generally refers to an iterative approach, that is calculated to offset the current point average, to move the point to its partial Mean-shift, and then as a new starting point and continue to move until the end to meet certain conditions.

However, after a long period of time has not Mean Shift unnoticed until 20 years later, that is, in 1995(Yizong Cheng et al, 1995), and Mean Shift on one of the important documents was published. In this important literature, Yizong Cheng of the basic Mean Shift Algorithm (Tan Tieniu et al, 2002) in the following two have done a promotion, first of all, the definition of a family of Yizong Cheng kernel, making shift (Liu Ruizhen et al, 2007;Yu Shiqi et al, 2007) with the sample points with different distance, the offset of the mean shift vector contribution (Wang Liang et al, 2002; Hu Weiming et al, 2002; Tan Tieniu et al, 2002) are also different, followed by Yizong Cheng also set a weight coefficient, making the importance of different sample points are not the same, which greatly expanded the scope of application of the Mean Shift.

Behind, this article will detail the basic idea of Mean Shift and its expansion, as well as the algorithm steps. Finally, this article will also be given in the video.

Mean Shift tracking of specific application (Liu Fuqiang et al, 2003; Li Lianye et al, 2005; Yan Hui et al, 2005; Zhu Weidong et al, 2005).

\section{Density Estimation}

Density estimation (Silverman B W et al, 1986) is from a group of unknown probability density distribution of the observations to estimate the probability density of its distribution to meet. There are usually two methods: parametric and non-parameter method. Parameter method is based on the assumption of data points is determined by our known distribution (such as Gaussian distribution) arising from, and then by the known distribution to approximate the requirements of the distribution; not known to find the distribution of its similar distribution, so that the probability estimation more accurate density distribution. Non-parametric density estimation method of many, such as the histogram method, the nearest method, kernel density estimation (Philippe Van Kerm et al, 2003.) methods, etc., which is the kernel density estimation of the most widely used technology, the following kernel density estimation is given the formula. In d dimensional space $\mathrm{Rd}$ in a given $\mathrm{n}$ data points (xi) $\mathrm{i}=1,2, \ldots, \mathrm{n}$, with $\mathrm{K}$ core density is estimated that the width of the window $h$. There are

$$
f(x)=\frac{1}{n h^{d}} \sum_{i=1}^{n} K\left(\frac{x-x_{i}}{h}\right)
$$

Which the kernel function $\mathrm{K}(\mathrm{x})$ must satisfy two conditions: $1, \mathrm{~K}$ as a symmetric probability density function (such as Gaussian density);

2, $\int_{0}^{\infty} K(x) d x=1$ 。 
For K (x), there are several different types of kernel functions to choose from, including Epanechnikov core(Scott D W et al, 1992) in the sense of minimum meanvariance optimal, one of the kernel function: In this paper, the experiment is carried out using core Epanechnikov density estimate of.

$$
K_{e}(x)= \begin{cases}\frac{1}{2} c_{d}^{-1}(d+2)\left(1-\|x\|^{2}\right) & \text { if }\|x\|<1 \\ 0 & \text { otherwise }\end{cases}
$$

Of which: cd as a unit the size of d-dimensional sphere, for example, c1 =2, c2 =, c3 = apparently, according to type (1) data can be a given probability density function estimation.

\section{Mean Shift Algorithm}

Mean Shift algorithm(Dorin Comaniciu et al;Peter Meer et al,1999) gradient method is iterative calculation using the probability density function of extreme points. Data have been obtained in accordance with the probability density function, its gradient is:

$$
\nabla f(x)=\frac{1}{n h^{d}} \sum_{i=1}^{n} \nabla K\left(\frac{x-x_{i}}{h}\right)
$$

Can be further transformed into:

$$
\nabla f(x)=\frac{n_{x}}{n\left(h^{d} c_{d}\right)} \frac{d+2}{h^{2}}\left(\frac{1}{n_{x}} \sum_{x_{i} \in S_{h}(x)}\left[x_{i}-x\right]\right)
$$

The scope of which Sh (x) for the radius of spherical super-h, volume of hdcd, their centers for $\mathrm{x}$ and include data points $\mathrm{nx}$.

Mean Shift Vector Mh (x) is defined as:

$$
M_{h}(x)=\frac{1}{n_{x}} \sum_{x_{i} \in S_{h}(x)}\left[x_{i}-x\right]=\frac{1}{n_{x}} \sum_{x_{i} \in S_{h}(x)} x_{i}-x
$$

The same type (4) can be written:

$$
M_{h}(x)=\frac{h^{2}}{d+2} \frac{\nabla f(x)}{f(x)}
$$

Style (5) and type (6) show that the local Mean Shift vector mean and the difference between window centers, point to the probability density function of the direction of the peak or valley points. The calculation steps are as follows:

First of all, to jump out of a cycle threshold e, from $x=x i, i=1, \ldots, n$ of departure: 1, calculating the Mean Shift Vector Mh (x); 2, will move the window by Mh (x) the size and the direction of movement; 3 , if $\mathrm{Mh}(\mathrm{x})$ than e, and then jump back to step (1) repeat; 4 , to the point of convergence Department reservations.

It can be seen, Mean Shift to speed up the increase in variable step size (down), and ultimately converge to the probability density function of the peak (valley) points. 


\section{Mean Shift Algorithm to Realize Thought a Experiment}

Mean Shift-based video tracking algorithm in the experiment, mainly through the tracking target on the current frame compared with the adjacent frames to determine the next frame the candidate for the current module or modules. Overall thinking is as follows:

Initialize the location of the current frame, and for the current Bhattacharyya coefficient (hereinafter referred to as bc coefficient) values. Calculate the location of new goals, new objectives and then update the location of the distribution, the experiment used to carry out core Epanechnikov kernel density estimation.

Then bc obtained updated coefficient, calculated the distance between two vectors, that is relatively current and updated bc coefficient, so as to come up with the movement more similar to the frame. Frame and set the current frame, so again the next candidate for the current module and the comparison module, until the track before the experiment to reach the frame default value, end of the code used to define the variables. Manually in the first frame (input file type) are identified, automatic target tracking. The experiments also support mouse control, which can be used in tracking the course of the mouse to control the suspension, termination or continuation of such.

In order to verify the previous chapter described the principle of Mean Shift tracking algorithm, this chapter will be in the video file in a cat on the track movement experiment. This video is downloaded from ww.snuffx.com. In this experiment the cat is a moving target, jumping from one side to the other side, moving target small and fast application of the agricultural surveillance video of intelligent tracking have a strong persuasive. Sequence in each frame size of $320 * 240$ in the first frame to be determined manually to determine the objectives of tracking, the algorithm in $\mathrm{VC}+$ +6.0 under development. Take the following video of the cat as an example, once every 10 frames as follows:

Tracking at the 4th frame

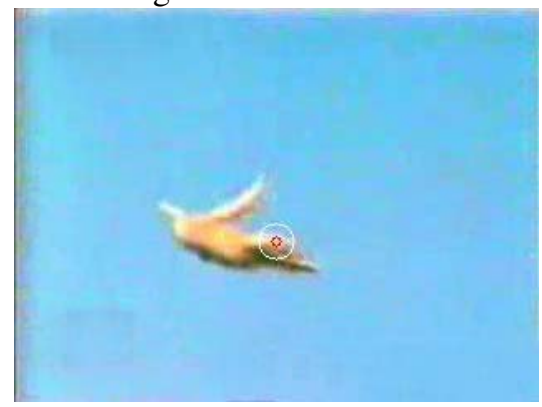

Tracking at the 24th frame

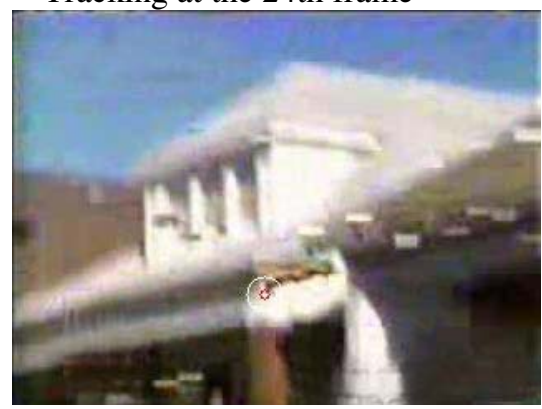

Tracking at the 14th frame

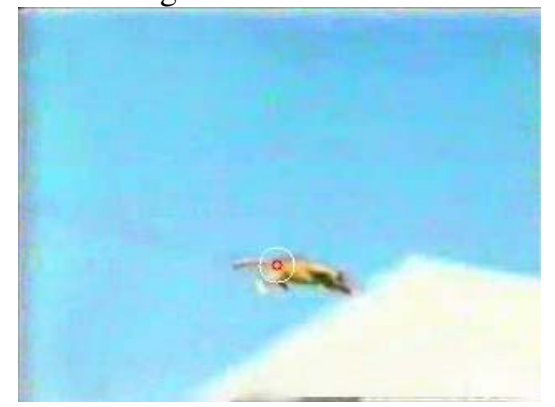

Tracking at the 34th frame

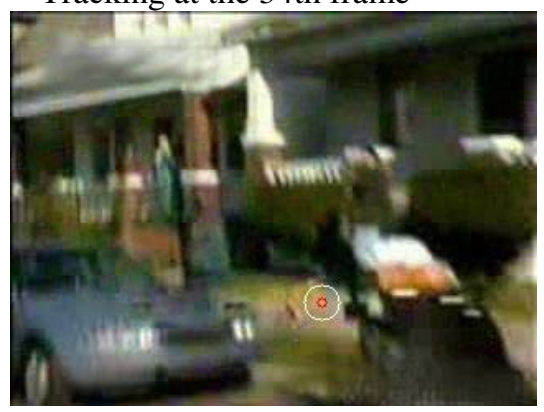




\section{Conclusion}

The agricultural tracking intelligent video surveillance is a new research topic, this article through experiments. Especially for the tracking algorithm analysis, Mean Shift Algorithm for the traditional model in the entire follow-up due to always remain the same cycle, positioning is not accurate enough, especially in the experiment to track moving objects in video action on the substantial difficulties in tracking moving target, this experiment a little Mean Shift Algorithm improved. Generally speaking, the more successful experiments and experiments show that the method of a small amount of real-time high, able to effectively track the moving target video.

\section{Acknowledgements}

Funding for this research was provided by Department of Science and Technology of Sichuan Province (P.R. China).

\section{References}

Silverman, B.W., et al.: The Estimation of the Gradient of a Density Function, with Applications in Pattern Recognition 48(9), 1022-1023 (1975)

Tieniu, T.: Intelligent Video Surveillance Technology. In: The First National Conference on intelligent visual surveillance, Beijing, vol. 38(11), pp. 1007-1011 (2002)

Scott, D.W.: Multivariate Density Estimation, vol. 56(11), pp. 1037-1044. Wiley, New York (1992)

Ruizhen, L., Shiqi, Y.: OpenCV Tutorial, 1st edn., pp. 357-392. Beijing University of Aeronautics and Astronautics Press, Beijing (2007)

Liu, F.-q.: Mathematics Video Surveillance System Development and Application, pp. 112-117. Mechanical Industry Press, Beijing (2003)

Lianye, L., Yan-hui, Z.W.: TV monitoring system to prevent invasion of design and construction technology, vol. 13(2), pp. 138-149. Publishing House of Electronics Industry, Beijing (2005)

The Estimation of the Gradient of a Density Function, with Applications in Pattern Recognition 38(5), 478-481 (1975)

Liang, W., Ming, H., Tieniu, T.: Visual analysis of human movement. A synthesis of Chinese Journal of Computers 25(3), 225-237 (2002)

Silverman, B.W.: Density Estimation for statistics and Data Analysis, vol. 48(9), pp. 698-701. Chapman and Hall, London (1986)

Van Kerm, P.: Adaptive kernel density estimation. In: 9th UK Stata Users meeting. In: 9th UK Stata Users meeting,Royal Statistical Society, London, May 19-20, vol. 26(10), pp. 147-151 (2003)

Comaniciu, D., Meer, P.: Mean Shift Analysis and Applications. In: Proceedings of the International Conference on Computer Vision, vol. 27(12), pp. 1191-1197 (1999) 